\title{
Hadronic parity violation in effective field theory
}

\author{
Matthias R. Schindler* ${ }^{* \dagger}$ \\ Department of Physics and Astronomy, University of South Carolina, Columbia, SC, USA \\ E-mail: mschindlemailbox.sc.edu
}

\begin{abstract}
Parity-violating interactions between nucleons are the manifestation of an interplay of strong and weak interactions between quarks in the nucleons. Because of the short range of the weak interactions, these parity-violating forces provide a unique probe of low-energy strong interactions. An ongoing experimental program is mapping out this weak component of the nuclear force in few-nucleon systems. Recent theoretical progress in analyzing and interpreting hadronic parity violation in such systems based on effective field theory methods is described. In particular, important information on parity-violating nucleon-nucleon interactions can be gained from an asymmetry in deuteron photodisintegration, which might be measurable at a future high-intensity photon source such as the proposed upgraded HIGS facility.
\end{abstract}

The 8th International Workshop on Chiral Dynamics, CD2015 ***

29 June 2015 - 03 July 2015

Pisa, Italy

\footnotetext{
${ }^{*}$ Speaker.

${ }^{\dagger}$ Support by the US Department of Energy under Grant No. DE-SC0010300 is gratefully acknowledged.
} 


\section{Introduction}

The forces between nucleons are dominated by the strong and electromagnetic interactions, both of which conserve parity. However, due to weak interactions between quarks, the forces between nucleons also have a parity-violating (PV) component. For reviews, see e.g. Refs. [1, 2, 3, 4, 5]. Typically, for the nucleon-nucleon (NN) interactions this PV part is expected to be suppressed by a factor of roughly $10^{-6}$ to $10^{-7}$ compared to the dominant parity-conserving (PC) part. Such small effects can be studied by considering observables that would vanish if parity was exactly conserved. Examples include induced polarizations as well as longitudinal and angular asymmetries when polarized beams or targets are considered. Under certain circumstances, such as closely spaced energy levels of opposite parity, the PV effects can be enhanced in heavy nuclei by several orders of magnitude, see e.g. Ref. [6]. However, the theoretical description and interpretation of these systems in terms of the underlying NN interactions is considerably more complex than for few-nucleon systems.

While we have a detailed understanding of the weak interactions at the quark level, their manifestation on the hadronic level is the result of an interplay between weak and the nonperturbative strong interactions that confine the quarks into hadrons. Therefore, the PV interactions between nucleons can be considered as a probe of nonperturbative QCD. In particular, because the range of the weak quark-quark interactions $(\sim 0.0002 \mathrm{fm})$ is much smaller than the typical size of a nucleon ( $\sim 1 \mathrm{fm}$ ), the PV nucleon forces are sensitive to quark-quark correlations inside the nucleon.

Traditionally, PV NN interactions have been parametrized in terms of single-meson exchanges [7], with a seminal analysis of the PV meson-nucleon couplings given in Ref. [8]. More recently, starting with the work of Ref. [9], a systematic approach in terms of effective field theories (EFTs) has been advocated and pursued. Depending on the relevant energies and momenta, two different approaches are being considered: at very low energies well below the pion mass, the so-called pionless $\mathrm{EFT}(\mathrm{EFT}(\not{t}))$ provides a description in terms of contact operators only involving nucleons and possibly external fields. At higher energies, pions can be resolved and have to be accounted for as dynamical degrees of freedom. This is systematically achieved in the so-called chiral EFT. One of the advantages of the EFT approaches is that they allow PC and PV interactions (as well as external currents) to be treated in the same systematic framework. In addition, EFTs in principle provide means to estimate theoretical uncertainties based on power counting arguments. For a recent review with a particular emphasis on the EFT formalism for PV interactions see Ref. [5].

In parallel with theoretical developments, significant improvements in high-intensity sources of neutron beams and in the control of systematics have made the detection of small PV effects in few-nucleon systems possible. Examples include the NPDGamma experiment at the Spallation Neutron Source (SNS) at Oak Ridge National Laboratory [10] and the measurement of the spin rotation angle in ${ }^{4} \mathrm{He}$ at the National Institute of Standards and Technology (NIST) [11].

\section{Parity violation in pionless EFT}

At energies well below the pion mass, pions (and heavier particles) cannot be resolved as dynamical degrees of freedom, and the interactions between nucleons can be described in terms of contact operators involving only nucleon fields and their coupling to external sources. In this 
pionless EFT the operators in the Lagrangian are ordered according to the number of derivatives and nucleon fields, and each operator is accompanied by a so-called low-energy constant (LEC) that encodes the short-distance physics. Observables are then calculated order-by-order in an expansion in a small parameter derived from a ratio of relevant scales. EFT $(\not t)$ has been very successfully applied for PC observables, with reviews given, e.g., in Refs. [12, 13, 14]. In the PC sector, the leading-order (LO) $\mathrm{NN}$ interaction terms can be written as

$$
\mathscr{L}_{P C}=-\frac{1}{8} \mathscr{C}_{0}^{\left({ }^{1} S_{0}\right)}\left(N^{T} \tau_{2} \tau_{a} \sigma_{2} N\right)^{\dagger}\left(N^{T} \tau_{2} \tau_{a} \sigma_{2} N\right)-\frac{1}{8} \mathscr{C}_{0}^{\left({ }^{3} S_{1}\right)}\left(N^{T} \tau_{2} \sigma_{2} \sigma_{i} N\right)^{\dagger}\left(N^{T} \tau_{2} \sigma_{2} \sigma_{i} N\right)+\ldots,
$$

where the $\vec{\sigma}(\vec{\tau})$ matrices act in spin (isospin) space.

There also exists a different formalism for $\operatorname{EFT}(\not t)$ (referred to as the dibaryon formalism in the following), in which two additional dynamical fields $d_{t}$ and $d_{i}$ with the quantum numbers of the real and virtual $\mathrm{NN}$ bound states in the ${ }^{3} S_{1}$ and ${ }^{1} S_{0}$ channels, respectively, are introduced $[15,16,17]$. These fields are auxiliary fields that can simplify calculations, in particular in the threenucleon sector, and $d_{t}$ can be used as an interpolating field for the deuteron. The LO Lagrangian corresponding to Eq. (2.1) and including the kinetic terms for the auxiliary fields is given by

$$
\begin{aligned}
\mathscr{L}_{P C}^{d}= & -\frac{y_{t}}{\sqrt{8}}\left[d_{t}^{i \dagger}\left(N^{T} \tau_{2} \sigma_{2} \sigma_{i} N\right)+\text { h.c. }\right]-\frac{y_{s}}{\sqrt{8}}\left[d_{s}^{a^{\dagger}}\left(N^{T} \tau_{2} \tau_{a} \sigma_{2} N\right)+\text { h.c. }\right] \\
& +d_{t}^{i \dagger}\left[\Delta_{t}-c_{0 t}\left(i D_{0}+\frac{\vec{D}^{2}}{4 M}+\frac{\gamma_{t}^{2}}{M}\right)\right] d_{t}^{i}+d_{s}^{A \dagger}\left[\Delta_{s}-c_{0 s}\left(i D_{0}+\frac{\vec{D}^{2}}{4 M}+\frac{\gamma_{s}^{2}}{M}\right)\right] d_{s}^{A},
\end{aligned}
$$

where $\gamma_{t}\left(\gamma_{s}\right)$ is the binding momentum of the real (virtual) bound state in the spin-triplet (spinsinglet) channel, $M$ the nucleon mass, and $y_{t / s}, \Delta_{t / s}$, and $c_{0 t / s}$ are LECs in the dibaryon formalism.

The PV Lagrangian in EFT( $\not$ ) at LO contains five independent terms, each with a corresponding LEC, and describes $S-P$ wave transitions [18, 19, 20],

$$
\begin{aligned}
\mathscr{L}_{P V}=- & {\left[\mathscr{C}^{\left({ }^{3} S_{1}-{ }^{1} P_{1}\right)}\left(N^{T} \sigma_{2} \vec{\sigma} \tau_{2} N\right)^{\dagger} \cdot\left(N^{T} \sigma_{2} \tau_{2} i \stackrel{\leftrightarrow}{D} N\right)\right.} \\
& +\mathscr{C}_{(\Delta I=0)}^{\left({ }^{1} S_{0}-{ }^{3} P_{0}\right)}\left(N^{T} \sigma_{2} \tau_{2} \vec{\tau} N\right)^{\dagger}\left(N^{T} \sigma_{2} \vec{\sigma} \cdot \tau_{2} \vec{\tau} i \stackrel{\leftrightarrow}{D} N\right) \\
& +\mathscr{C}_{(\Delta I=1)}^{\left({ }^{1} S_{0}-{ }^{3} P_{0}\right)} \varepsilon^{3 a b}\left(N^{T} \sigma_{2} \tau_{2} \tau^{a} N\right)^{\dagger}\left(N^{T} \sigma_{2} \vec{\sigma} \cdot \tau_{2} \tau^{b} \stackrel{\leftrightarrow}{D} N\right) \\
& +\mathscr{C}_{(\Delta I=2)}^{\left({ }^{1} S_{0}-{ }^{3} P_{0}\right)} \mathscr{I}^{a b}\left(N^{T} \sigma_{2} \tau_{2} \tau^{a} N\right)^{\dagger}\left(N^{T} \sigma_{2} \vec{\sigma} \cdot \tau_{2} \tau^{b} \stackrel{\leftrightarrow}{D} N\right) \\
& \left.+\mathscr{C}^{\left({ }^{3} S_{1}-{ }^{3} P_{1}\right)} \varepsilon^{i j k}\left(N^{T} \sigma_{2} \sigma^{i} \tau_{2} N\right)^{\dagger}\left(N^{T} \sigma_{2} \sigma^{k} \tau_{2} \tau_{3} \stackrel{\leftrightarrow}{D^{j}} N\right)\right]+ \text { h.c. },
\end{aligned}
$$

where $a \mathscr{O} \stackrel{\leftrightarrow}{D} b=a \mathscr{O} \vec{D} b-(\vec{D} a) \mathscr{O} b$ with $\mathscr{O}$ some spin-isospin-operator, and $\mathscr{I}=\operatorname{diag}(1,1,-2)$. Analogously, in the dibaryon formalism it is given by [21]

$$
\begin{aligned}
\mathscr{L}_{P V}= & -\left[g^{\left({ }^{3} S_{1}-{ }^{1} P_{1}\right)} d_{t}^{i \dagger}\left(N^{T} \sigma_{2} \tau_{2} \stackrel{\leftrightarrow}{D_{i}} N\right)\right. \\
& +g_{(\Delta I=0)}^{\left({ }^{1} S_{0}-{ }^{3} P_{0}\right)} d_{s}^{A \dagger}\left(N^{T} \sigma_{2} \vec{\sigma} \cdot \tau_{2} \tau_{A} i \stackrel{\leftrightarrow}{D} N\right) \\
& +g_{(\Delta I=1)}^{\left({ }^{1} S_{0}-{ }^{3} P_{0}\right)} \varepsilon^{3 A B} d_{s}^{A \dagger}\left(N^{T} \sigma_{2} \vec{\sigma} \cdot \tau_{2} \tau^{B} \stackrel{\leftrightarrow}{D} N\right) \\
& +g_{(\Delta I=2)}^{\left({ }^{(} S_{0}-{ }^{3} P_{0}\right)} \mathscr{I}^{A B} d_{s}^{A \dagger}\left(N^{T} \sigma_{2} \vec{\sigma} \cdot \tau_{2} \tau^{B} i \stackrel{\leftrightarrow}{D} N\right) \\
& \left.+g^{\left({ }^{3} S_{1}-{ }^{3} P_{1}\right)} \varepsilon^{i j k} d_{t}^{i \dagger}\left(N^{T} \sigma_{2} \sigma^{k} \tau_{2} \tau_{3} \stackrel{\leftrightarrow}{D}{ }^{j} N\right)\right]+ \text { h.c. }
\end{aligned}
$$


The five LECs $\mathscr{C}^{(X-Y)}\left(g^{(X-Y)}\right.$ in the dibaryon formalism) encode the short-distance physics that results in PV interactions between nucleons. They cannot be determined within the EFT framework. In principle, they can be calculated in the standard model which, however, involves a nonperturbative QCD calculation. So far, no such calculation of these LECs has been performed. Alternatively, given sufficient experimental information, they can be determined from data and then in turn can serve as targets that future calculations will have to reproduce. The Lagrangians above can be considered the field theoretic equivalent of the formalism of Refs. [22, 23].

A number of observables have been calculated in the EFT( $\not$ ) framework. The PV longitudinal asymmetry in $\vec{N} N$ scattering is given by

$$
A_{L}=\frac{\sigma_{+}-\sigma_{-}}{\sigma_{+}+\sigma_{-}}
$$

where $\sigma_{ \pm}$is the total cross section for scattering with a beam of helicity \pm 1 . In the conventions of Eq. (2.4), the LO results are [20]

$$
\begin{aligned}
A_{L}^{n n}= & -\sqrt{\frac{32 M}{\pi}} p\left(g_{(\Delta I=0)}^{\left({ }^{1} S_{0}-{ }^{3} P_{0}\right)}-g_{(\Delta I=1)}^{\left({ }^{1} S_{0}-{ }^{3} P_{0}\right)}+g_{(\Delta I=2)}^{\left({ }^{1} S_{0}-{ }^{3} P_{0}\right)}\right), \\
A_{L}^{p p}= & -\sqrt{\frac{32 M}{\pi}} p\left(g_{(\Delta I=0)}^{\left({ }^{1} S_{0}-{ }^{3} P_{0}\right)}+g_{(\Delta I=1)}^{\left({ }^{1} S_{0}-{ }^{3} P_{0}\right)}+g_{(\Delta I=2)}^{\left({ }^{1} S_{0}-{ }^{3} P_{0}\right)}\right), \\
A_{L}^{n p}= & -\sqrt{\frac{32 M}{\pi}} p \frac{\frac{d \sigma^{1} S_{0}}{d \Omega}}{\frac{d \sigma^{1} S_{0}}{d \Omega}+3 \frac{d \sigma^{3} S_{1}}{d \Omega}}\left(g_{(\Delta I=0)}^{\left({ }^{1} S_{0}-{ }^{3} P_{0}\right)}-2 g_{(\Delta I=2)}^{\left({ }^{1} S_{0}-{ }^{3} P_{0}\right)}\right) \\
& -\sqrt{\frac{32 M}{\pi}} p \frac{\frac{d \sigma^{3} S_{1}}{d \Omega}}{\frac{d \sigma^{1} S_{0}}{d \Omega}+3 \frac{d \sigma^{3} S_{1}}{d \Omega}}\left(g^{\left({ }^{3} S_{1}-{ }^{1} P_{1}\right)}+2 g^{\left({ }^{3} S_{1}-{ }^{3} P_{1}\right)}\right),
\end{aligned}
$$

where $p$ is the nucleon momentum in the center-of-mass frame. Coulomb effects are neglected in the $\vec{p} p$ case, but only amount to an approximately 3\% correction [20] at the lowest energy at which $A_{L}$ has been measured [24].

While it is currently not feasible to measure the longitudinal asymmetry for the $n p$ case, the corresponding scattering amplitude is related to a different PV observable. If a perpendicularly polarized beam traverses an unpolarized target, PV interactions result in a rotation of the spin about the axis of propagation. For a neutron beam and a proton target, the NLO result for the spin rotation angle per unit length is [25]

$$
\frac{1}{\rho} \frac{d \phi_{\mathrm{PV}}^{n p}}{d l}=4 \sqrt{2 \pi M}\left(\frac{2 g^{\left({ }^{3} S_{1}-{ }^{3} P_{1}\right)}+g^{\left({ }^{3} S_{1}-{ }^{1} P_{1}\right)}}{\gamma_{t}} \frac{Z_{t}+1}{2}+\frac{g_{(\Delta I=0)}^{\left({ }^{1} S_{0}-{ }^{3} P_{0}\right)}-2 g_{(\Delta I=2)}^{\left({ }^{1} S_{0}-{ }^{3} P_{0}\right)}}{\gamma_{s}} \frac{Z_{s}+1}{2}\right),
$$

where $\rho$ is the target density, $Z_{t / s}=\left(1-\gamma_{t / s} r_{t / s}\right)^{-1}$, and $r_{t / s}$ denotes the effective range in the spin-triplet/spin-singlet channel.

Measurement of the PV angular asymmetry $A_{\gamma}$ in the radiative capture of polarized neutrons on protons $(\vec{n} p \rightarrow d \gamma)$ has been a longstanding goal; the NPDGamma collaboration at the SNS has finished data taking, with results eagerly awaited to be announced (see the contribution by L. Barrón Palos). The LO EFT( $\not$ ) result is $[26,21]$

$$
A_{\gamma}=\frac{4}{3} \sqrt{\frac{2}{\pi}} \frac{M^{\frac{3}{2}}}{\kappa_{1}\left(1-\gamma_{t} a_{S}\right)} g^{\left({ }^{3} S_{1}-{ }^{3} P_{1}\right)},
$$


with $a_{s}$ the scattering length in the ${ }^{1} S_{0}$ channel and $\kappa_{1}$ the isovector nucleon anomalous magnetic moment.

The radiative capture of unpolarized neutrons on an unpolarized proton target provides an independent and complementary PV observable in the induced circular polarization $P_{\gamma}$ of the outgoing photon $(n p \rightarrow d \vec{\gamma})$. It is identical to the PV asymmetry $A_{L}^{\gamma}$ in the deuteron breakup reaction with polarized photons $(\vec{\gamma} d \rightarrow n p)$ for reversed kinematics,

$$
A_{L}^{\gamma}=\frac{\sigma_{+}-\sigma_{-}}{\sigma_{+}+\sigma_{-}}
$$

where $\sigma_{ \pm}$is the total cross section for photons with helicity \pm 1 . The small expected size of the asymmetry places stringent requirements on the beam intensity and control of systematic effects for a future experiment. The possibility of such a measurement has been under consideration at the High Intensity Gamma-Ray Source at the Triangle Universities Nuclear Laboratory. A NLO calculation of the asymmetry and its energy dependence was performed in Ref. [27]. The result for $A_{L}^{\gamma}$ is shown in Fig. 1. Because the values of the LECs are currently not known, three different model estimates are used for illustrative purposes. Given the uncertainty in the values, these results should only be considered as an order-of-magnitude estimate. Because of the denominator in Eq. (2.10),

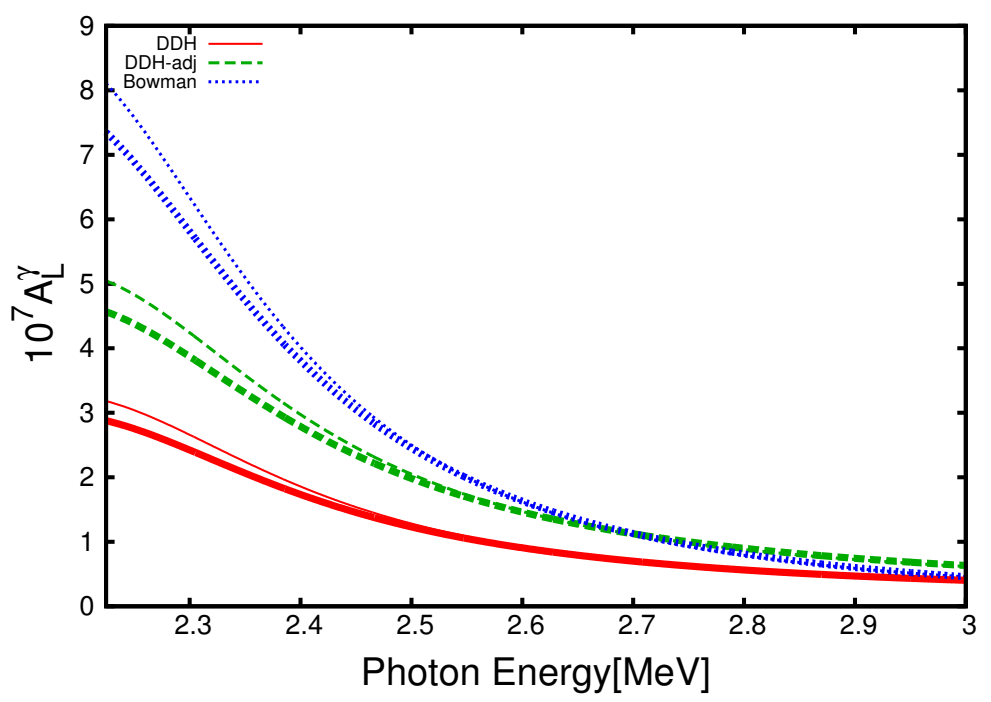

Figure 1: Energy dependence of the PV asymmetry $A_{L}^{\gamma}$ for various LEC estimates from Ref. [27]. The thin and thick lines correspond to different $\mathrm{EFT}(\pi)$ parametrizations and give an indication of the theoretical uncertainty.

the asymmetry is maximum at threshold. In order to find an energy range at which to perform the experiment, a simplistic figure of merit was considered in Ref. [27],

$$
f=\left(A_{L}^{\gamma}\right)^{2} \times \sigma(\gamma d \rightarrow n p) .
$$

For the LEC estimates used in this work, the figure of merit has its maximum in the energy range $2.259 \mathrm{MeV}<k<2.264 \mathrm{MeV}$.

In order to determine all five LO LECs from experimental data, systems involving more than two nucleons become important. In the PC sector of three-nucleon systems it was found that while a 


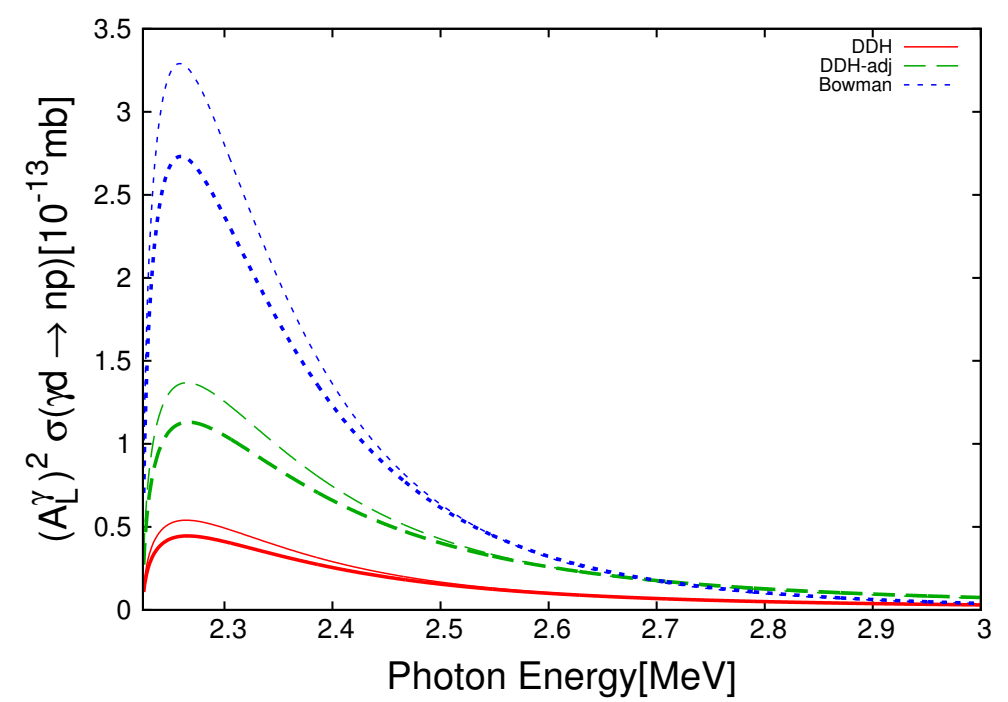

Figure 2: Figure of merit $f$ for various LEC estimates from Ref. [27]. The thin and thick lines correspond to different $\operatorname{EFT}(\pi)$ parametrizations and give an indication of the theoretical uncertainty.

straightforward application of the power counting rules does not predict three-nucleon interactions at LO, an unphysical cutoff dependence in the LO scattering amplitude for spin-doublet neutrondeuteron scattering can be removed by the introduction of a three-nucleon term already at this order [28]. The corresponding LEC can be determined from experimental input, e.g. the neutrondeuteron scattering length or a three-body binding energy. A similar enhancement of three-nucleon forces in the PV sector would be problematic given the scarcity of and complexity in obtaining sufficient experimental data. The analysis of Ref. [29] showed that no such enhancement of a PV three-nucleon contact term occurs at LO and NLO in nucleon-deuteron scattering. This means that up to an accuracy of approximately $10 \%$, it is possible to analyze few-nucleon observables in terms of PV two-nucleon interactions.

Analogously to the case of $\vec{n} p$ scattering, the $\vec{n} d$ scattering amplitude is related to the spin rotation angle of a polarized neutron beam traversing a deuteron target. The NLO expression for the rotation angle is [25]

$$
\begin{aligned}
\frac{1}{\rho} \frac{d \phi_{\mathrm{PV}}^{n d}}{d l}=( & {[16 \pm 1.6] g^{\left({ }^{3} S_{1}-{ }^{1} P_{1}\right)}+[34 \pm 3.4] g^{\left({ }^{3} S_{1}-{ }^{3} P_{1}\right)} } \\
& \left.+[4.6 \pm 1.0]\left(3 g_{(\Delta I=0)}^{\left({ }^{1} S_{0}-{ }^{3} P_{0}\right)}-2 g_{(\Delta I=1)}^{\left({ }^{1} S_{0}-{ }^{3} P_{0}\right)}\right)\right) \frac{\mathrm{rad}}{\mathrm{MeV}^{\frac{1}{2}}} .
\end{aligned}
$$

Here, the theoretical uncertainties correspond to the most conservative of three different estimates based on power counting, cutoff dependence in the corresponding integral equations, and different ways to determine the PC three-nucleon LEC.

\section{Parity violation in chiral EFT}

At higher energies the pion has to be treated as a dynamical degree of freedom. Using the chiral perturbation theory formalism, the PV pion-nucleon Lagrangian up to terms including a 
single pion derivative was constructed in Ref. [9]. The leading pion-nucleon interaction term is given by

$$
\mathscr{L}_{P V}^{\pi N N}=-i h_{\pi N N}^{1}\left(\bar{p} \pi^{+} n-\bar{n} \pi^{-} p\right)+\ldots
$$

The ellipsis stands for further terms containing additional pion and/or external fields. No measurement of a PV observable in the single-nucleon sector exists; however, PV asymmetries in Compton scattering [30, 31] and pion production [32, 33], as well as the nucleon anapole moment [34, 35, 36] have been considered theoretically.

The Lagrangian of Eq. (3.1) can be combined with the corresponding PC pion-nucleon Lagrangian to derive a PV NN potential [18]. The leading one-pion-exchange potential takes the form

$$
V_{P V}=-i \frac{g_{A} h_{\pi N N}^{1}}{2 \sqrt{2} F^{2}} \frac{\left(\vec{\sigma}_{1}+\vec{\sigma}_{2}\right) \cdot \vec{q}}{\vec{q}^{2}+m_{\pi}^{2}}\left(\vec{\tau}_{1} \times \vec{\tau}_{2}\right)_{z}+\ldots
$$

where $g_{A}$ is the axial-vector coupling constant, $F$ denotes the pion decay constant, and the ellipsis stands for higher-order contributions. Power counting indicates that this is the complete PV potential at $\mathrm{LO}$ and that a potential derived from contact terms equivalent to those of Eq. (2.3) contributes at NLO. For a detailed discussion of the PV potential see Refs. [18, 37, 38]. However, this counting is based on the naturalness assumption for the involved LECs. There are some theoretical indications that $h_{\pi N N}^{1}$ might be smaller than expected [39, 40, 41], in agreement with a determination from ${ }^{18} \mathrm{~F}$ [42]. This would indicate that the pion-exchange contribution is less significant than expected and that contact terms might play a more important role. The results from the NPDGamma collaboration are expected to make a large impact on this question. $h_{\pi N N}^{1}$ has also been the target of a pioneering lattice QCD study, which finds agreement with small values of $h_{\pi N N}^{1}$ [43].

The PV chiral potential has been used in the determination of PV observables from reactions such as $\vec{p} p$ scattering, radiative neutron capture on the proton, neutron spin rotation in proton and deuterium targets, as well as the charge-exchange reaction ${ }^{3} \mathrm{He}(\vec{n}, p)^{3} \mathrm{H}[44,37,45]$.

\section{Conclusions}

Arising from an interplay of short-range weak and nonperturbative strong interactions, hadronic parity violation provides a unique probe of low-energy QCD. While experimentally more accessible in complex nuclei, the difficulties in the theoretical analysis and interpretation of the corresponding observables in terms of nucleon-nucleon interactions has motivated a program to map out hadronic parity violation in few-nucleon systems. Recent developments have focused on the use of EFTs to describe PV NN interactions. At very low energies, $\mathrm{EFT}(\not{t})$ at LO parametrizes these interactions in terms of five contact terms, each with a corresponding LEC. At higher energies, pions can be introduced as dynamical degrees of freedom in a chiral EFT. The theory is then described in terms of six LECs, one of which is a PV pion-nucleon coupling. Determination of these LECs from a suite of experiments will provide a nontrivial target that any calculation in terms of standard model degrees of freedom will have to reproduce. The combination of continuing experimental developments, such as high-intensity sources of neutron and photon beams, further EFT calculations in few-nucleon systems, as well as future lattice QCD calculations of PV LECs offers the opportunity to significantly improve our understanding of hadronic parity violation. 


\section{Acknowledgments}

I thank H.W. Grießhammer, D.R. Phillips, W.M. Snow, R.P. Springer, and J. Vanasse for interesting and stimulating discussions.

\section{References}

[1] E. G. Adelberger and W. C. Haxton, Parity Violation in the Nucleon-Nucleon Interaction, Ann. Rev. Nucl. Part. Sci. 35 (1985) 501-558.

[2] W. Haeberli and B. R. Holstein, Parity Violation and the Nucleon-Nucleon System, In *Haxton, W.C. and Henley, E.M. (eds.): Symmetries And Fundamental Interactions In Nuclei* (1995) 17-66. , [nucl-th/9510062].

[3] M. J. Ramsey-Musolf and S. A. Page, Hadronic parity violation: A new view through the looking glass, Ann. Rev. Nucl. Part. Sci. 56 (2006) 1-52, [hep-ph / 0601127$].$

[4] W. C. Haxton and B. R. Holstein, Hadronic Parity Violation, Prog.Part.Nucl.Phys. 71 (2013) 185-203, [arXiv:1303.4132].

[5] M. R. Schindler and R. P. Springer, The Theory of Parity Violation in Few-Nucleon Systems, Prog.Part.Nucl.Phys. 72 (2013) 1-43, [arXiv:1305.4190].

[6] C. Bowman, J. Bowman, and V. Yuan, Parity violation in the 0.734-eV neutron resonance in ${ }^{139} \mathrm{La}$, Phys.Rev. C39 (1989) 1721-1724.

[7] F. C. Michel, Parity Nonconservation in Nuclei, Phys. Rev. 133 (1964) B329-B349.

[8] B. Desplanques, J. F. Donoghue, and B. R. Holstein, Unified Treatment of the Parity Violating Nuclear Force, Annals Phys. 124 (1980) 449.

[9] D. B. Kaplan and M. J. Savage, An Analysis of parity violating pion - nucleon couplings, Nucl. Phys. A556 (1993) 653-671. [Erratum-ibid. A 570, 833 (1994)] [Erratum-ibid. A 580, 679 (1994)].

[10] M. T. Gericke, R. Alarcon, S. Balascuta, L. Barron-Palos, C. Blessinger, et al., Measurement of parity-violating gamma-ray asymmetry in the capture of polarized cold neutrons on protons, Phys.Rev. C83 (2011) 015505.

[11] W. M. Snow, C. D. Bass, T. D. Bass, B. E. Crawford, K. Gan, et al., Upper bound on parity-violating neutron spin rotation in He-4, Phys.Rev. $\mathbf{C 8 3}$ (2011) 022501.

[12] S. R. Beane, P. F. Bedaque, W. C. Haxton, D. R. Phillips, and M. J. Savage, From hadrons to nuclei: Crossing the border, In *Shifman, M. (ed.): At the frontier of particle physics, vol. $1 *(2000)$ 133-269. , [nucl-th/0008064].

[13] P. F. Bedaque and U. van Kolck, Effective field theory for few-nucleon systems, Ann. Rev. Nucl. Part. Sci. 52 (2002) 339-396, [nucl-th/0203055].

[14] L. Platter, Low-Energy Universality in Atomic and Nuclear Physics, Few Body Syst. 46 (2009) 139-171, [arXiv:0904.2227].

[15] D. B. Kaplan, More effective field theory for nonrelativistic scattering, Nucl. Phys. B494 (1997) 471-484, [nucl-th/9610052].

[16] P. F. Bedaque and U. van Kolck, Nucleon deuteron scattering from an effective field theory, Phys.Lett. B428 (1998) 221-226, [nucl-th/9710073]. 
[17] S. R. Beane and M. J. Savage, Rearranging pionless effective field theory, Nucl. Phys. A694 (2001) 511-524, [nucl-th/0011067].

[18] S.-L. Zhu, C. M. Maekawa, B. R. Holstein, M. J. Ramsey-Musolf, and U. van Kolck, Nuclear parity-violation in effective field theory, Nucl.Phys. A748 (2005) 435-498, [nucl-th/ 0407087 ].

[19] L. Girlanda, On a redundancy in the parity-violating 2-nucleon contact Lagrangian, Phys. Rev. C77 (2008) 067001, [arXiv: 0804.0772 ].

[20] D. R. Phillips, M. R. Schindler, and R. P. Springer, An effective-field-theory analysis of low-energy parity-violation in nucleon-nucleon scattering, Nucl. Phys. A822 (2009) 1-19, [arXiv:0812.2073].

[21] M. R. Schindler and R. P. Springer, Two parity violating asymmetries from $n p \rightarrow d \gamma$ in pionless effective field theories, Nucl. Phys. A846 (2010) 51-62, [arXiv: 0907.5358 ].

[22] G. S. Danilov, Circular polarization of $\gamma$ quanta in absorption of neutrons by protons and isotopic structure of weak interactions, Phys.Lett. 18 (1965), no. 1 40-41.

[23] G. S. Danilov, Dispersion approach to the investigation of the weak nucleon-nucleon interaction, Phys.Lett. B35 (1971) 579-580.

[24] P. D. Eversheim, W. Schmitt, S. E. Kuhn, F. Hinterberger, P. von Rossen, et al., Parity violation in proton proton scattering at 13.6-MeV, Phys.Lett. B256 (1991) 11-14.

[25] H. W. Grießhammer, M. R. Schindler, and R. P. Springer, Parity-violating neutron spin rotation in hydrogen and deuterium, Eur.Phys.J. A48 (2012) 7, [arXiv:1109.5667].

[26] M. J. Savage, Parity Violation in Low-Energy $n p \rightarrow d$ gamma and the Deuteron Anapole Moment, Nucl. Phys. A695 (2001) 365-373, [nucl-th/ 012043$].$

[27] J. Vanasse and M. R. Schindler, Energy dependence of the parity-violating asymmetry of circularly polarized photons in $d \vec{\gamma} \rightarrow n p$ in pionless effective field theory, Phys. Rev. C90 (2014), no. 4 044001, [arXiv:1404.0658].

[28] P. F. Bedaque, H. W. Hammer, and U. van Kolck, Effective Theory of the Triton, Nucl. Phys. A676 (2000) 357-370, [nucl-th/9906032].

[29] H. W. Grießhammer and M. R. Schindler, On Parity-Violating Three-Nucleon Interactions and the Predictive Power of Few-Nucleon EFT at Very Low Energies, Eur. Phys. J. A46 (2010) 73-83, [arXiv:1007.0734].

[30] P. F. Bedaque and M. J. Savage, Parity violation in gamma polarized-p Compton scattering, Phys. Rev. C62 (2000) 018501, [nucl-th/ 9909055 ].

[31] J.-W. Chen, T. D. Cohen, and C. W. Kao, Parity violation in polarized-gamma proton Compton scattering, Phys. Rev. C64 (2001) 055206, [nucl-th/ 0009031 ].

[32] J.-W. Chen and X.-D. Ji, Measuring the P-odd pion-nucleon coupling $h_{\pi N N}^{(1)}$ in $\pi^{+}$photoproton production near the threshold, Phys. Rev. Lett. 86 (2001) 4239-4242, [hep-ph/ 0011230$].$

[33] S.-L. Zhu, S. Puglia, B. R. Holstein, and M. J. Ramsey-Musolf, Subleading corrections to parity violating pion photoproduction, Phys. Rev. C64 (2001) 035502, [hep-ph/ 0012253 ].

[34] M. J. Savage and R. P. Springer, The Anapole form-factor of the deuteron, Nucl.Phys. $\mathbf{A 6 8 6}$ (2001) 413-428, [nucl-th/9907069].

[35] C. M. Maekawa, J. S. Veiga, and U. van Kolck, The Nucleon anapole form-factor in chiral perturbation theory to subleading order, Phys. Lett. B488 (2000) 167-174, [hep-ph/ 0006181$].$ 
[36] C. M. Maekawa and U. van Kolck, The Anapole form-factor of the nucleon, Phys. Lett. B478 (2000) 73-78, [hep-ph/0006161].

[37] M. Viviani, A. Baroni, L. Girlanda, A. Kievsky, L. E. Marcucci, and R. Schiavilla, Chiral effective field theory analysis of hadronic parity violation in few-nucleon systems, Phys. Rev. C89 (2014), no. 6 064004, [arXiv:1403.2267].

[38] J. de Vries, N. Li, U.-G. Meißner, N. Kaiser, X. H. Liu, and S. L. Zhu, A study of the parity-odd nucleon-nucleon potential, Eur. Phys. J. A50 (2014) 108, [arXiv: 1404.1576 ].

[39] N. Kaiser and U.-G. Meißner, Novel Calculation of Weak Meson Nucleon Couplings, Nucl.Phys. A499 (1989) 699.

[40] U.-G. Meißner and H. Weigel, The Parity violating pion nucleon coupling constant from a realistic three flavor Skyrme model, Phys.Lett. B447 (1999) 1-7, [nucl-th/980 0 38].

[41] D. R. Phillips, D. Samart, and C. Schat, Parity-Violating Nucleon-Nucleon Force in the $1 / N_{c}$ Expansion, Phys. Rev. Lett. 114 (2015), no. 6 062301, [arXiv:1410.1157].

[42] W. Haxton, Parity Nonconservation in F-18 and Meson Exchange Contributions to the Axial Charge Operator, Phys.Rev.Lett. 46 (1981) 698.

[43] J. Wasem, Lattice QCD Calculation of Nuclear Parity Violation, Phys.Rev. C85 (2012) 022501, [arXiv:1108.1151].

[44] J. de Vries, U.-G. Meißner, E. Epelbaum, and N. Kaiser, Parity violation in proton-proton scattering from chiral effective field theory, Eur. Phys. J. A49 (2013) 149, [arXiv:1309.4711].

[45] J. de Vries, N. Li, U.-G. Meißner, A. Nogga, E. Epelbaum, and N. Kaiser, Parity violation in neutron capture on the proton: Determining the weak pion-nucleon coupling, Phys. Lett. B747 (2015) 299-304, [arXiv:1501.01832]. 